\title{
Functionalized Transparent Surfaces with Enhanced Self-Cleaning against Ink Aerosol Contamination
}

\author{
Mehmet A. Noyan, Denis Guilhot, and Valerio Pruneri*
}

During operation of a standard commercial inkjet printer, suspended ink particles form an ink aerosol inside the printing chamber that can cause serious malfunctions, including contamination of the transparent window of the printhead position calibration optical sensors. In this work, transparent conducting film (TCF) and surface functionalization through self-assembled monolayer (SAM) are proposed and investigated to repel ink aerosol deposition on a transparent surface and to reduce its adverse effects. The results show that the combination of the Joule heating effect induced by applying an electrical current to the TCF and hydrophobic property of the SAM reduces transmittance loss from an average of $10 \%$ to less than $1.5 \%$. Correspondingly, the area of the surface covered by ink decreases from $45.62 \% \pm 6.15 \%$ to $1.71 \% \pm 0.25 \%$. The preliminary results are obtained with glass substrates and subsequently extended to the plastic window of a commercial inkjet printer calibration sensor, thus demonstrating the potential of the proposed approach to reduce aerosol contamination in real applications.

\section{Introduction}

Aerosol is the name given to small solid or liquid ink droplets suspended in a gas, usually air. For example, fog is a natural aerosol made of small water droplets. Ink aerosol forms inside inkjet printers, the most common printer type on the market today. Inkjet printing is used extensively for printing text and images onto substrates, but it is also used for printing electronics, optical devices, and biological arrays. ${ }^{[1-5]}$ The basic principle of inkjet printing is to fire ink droplets generated through the application of a pulse of pressure, through a set of nozzles and onto a substrate (paper, plastic, etc.), to create the pixels of the intended image. ${ }^{[6]}$ The ink is made of a colorant (e.g., pigment, dye) and a vehicle (e.g., water, organic solvent), and the ink droplets in the aerosol can originate from two main mechanisms: (i) dispersion into the surrounding air of larger printing droplets flying from the printhead to the paper, and (ii) droplets themselves, when tiny enough to get incorporated into the aerosol.

\footnotetext{
M. A. Noyan, Dr. D. Guilhot, Prof. V. Pruneri

ICFO-Institut de Ciències Fotóniques

The Barcelona Institute of Science and Technology

Av. Carl Friedrich Gauss, 3, 08860 Castelldefels, Spain

E-mail: valerio.pruneri@icfo.eu

Prof. V. Pruneri

ICREA-Institució Catalana de Recerca i Estudis Avançats

Passeig Lluís Companys, 23

08010 Barcelona, Spain
}

DOI: 10.1002/admt.201600113
Notably, to prevent ink drying on the nozzles, printheads clean them by spraying excess ink, collected into a separate component called spittoon. This action is an important source of aerosol generation. The aerosol generation issue is more critical in large format printers and the occurrence of aerosol-related failures increased, seeing as these are high performance machines consuming significant amounts of ink compared to desktop printers.

Ink aerosol can accumulate on the mechanical components which may get in contact with the printing media, hence decreasing the quality of the image, and on encoder strips, causing false readings and eventually failures. Still, the most significant detrimental effect caused by ink aerosol is the contamination of the transparent windows of optical sensors, one of which is a multipurpose sensor used for calibration of the printhead position, color, and media advanced systems, shown in Figure S1 of the Supporting Information. Aerosol deposition on this photodetector protection window reduces transparency and prevents the light from reaching the sensor, which affects its functional performance.

Several approaches have been proposed to reduce the effects associated with ink aerosol contamination, for example, optimizing designs and algorithms to reduce its generation ${ }^{[7]}$ or increase its collection while it flies around inside the printer chamber. ${ }^{[8]}$ However, these methods are holistic, i.e., they reduce aerosol contamination on critical parts as well as noncritical parts. An approach addressing the issue of aerosol contamination on specific parts would be energy saving. In this paper, we aim at designing and developing functionalized surfaces that can prevent deposition of ink aerosol on transparent substrates and reduce contamination of critical parts in inkjet printers.

Figure 1 depicts the proposed surface functionalization approaches. First, transparent conducting films (TCFs) were used in order to heat up the surface of a transparent substrate using electrical currents (Joule effect). In this way, liquid ink vehicle droplets that get in contact with the surface evaporate. Second, we applied low surface tension self-assembled monolayer (SAM) to achieve hydrophobic surfaces. Such surfaces have large contact angles that prevent the spreading of the droplets, thus reducing the area shadowed by the ink aerosol and hence increasing wavelength dependent transparency. Moreover, vehicle and colorant slippage is favored, thanks to the lower sliding angle. While it is known that functionalized surfaces with TCF or SAM can repel liquids, ${ }^{[9]}$ they have never been applied to ink aerosol and in a demanding environment 


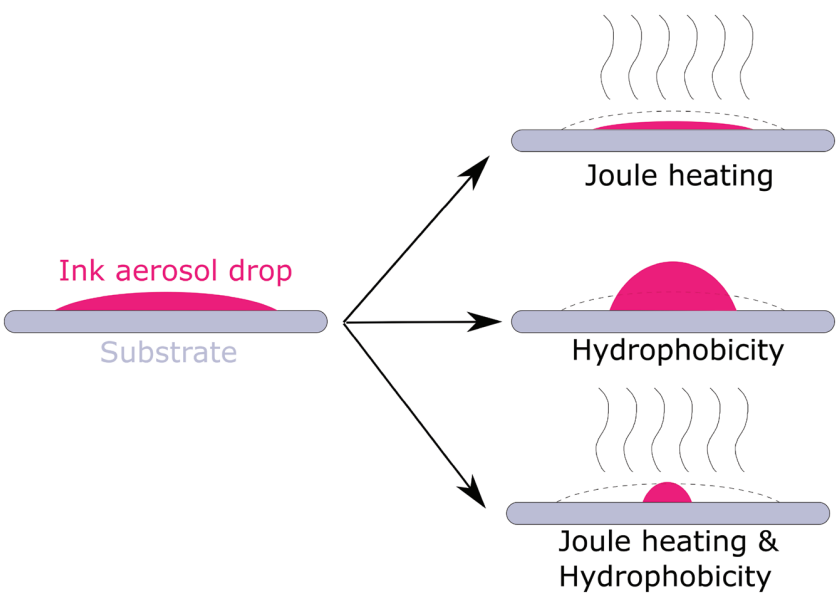

Figure 1. A simple schematic visualization of the concept demonstrated in this paper.

such as that of a high-throughput printer. Third, we combine for the first time Joule heating and hydrophobicity to further enhance the self-cleaning properties provided by the two methods separately. Combination of TCF and SAM was reported before, but the aim of that work was to protect TCF from environmental degradation using SAM.[10] Heating or self-cleaning was not involved.

Our results show that by combining Joule heating and hydrophobicity, it is possible to decrease aerosol contamination on glass substrates significantly. The strong contamination reduction effect was also confirmed for the plastic transparent window of the calibration sensor of a commercial inkjet printer.

\section{Results and Discussion}

Before the experiments with ink aerosol, Joule heating and hydrophobicity were characterized. For Joule heating, electrical current was applied to the sample using TCF. To maintain the temperature of the sample between 80 and $85^{\circ} \mathrm{C}$, the necessary power per unit surface area was measured to be $0.64 \mathrm{~W} \mathrm{~cm}^{-2}$. It took less than $3 \mathrm{~min}$ to reach the desired temperature. When the temperature was stabilized, heat distribution over the sample surface was observed to be uniform. More details can be seen in Figure S2 (Supporting information) where evolution of temperature over time was plotted upon the application of power and an IR image shows the heat distribution. For hydrophobicity due to SAM, contact angle (CA) and sliding

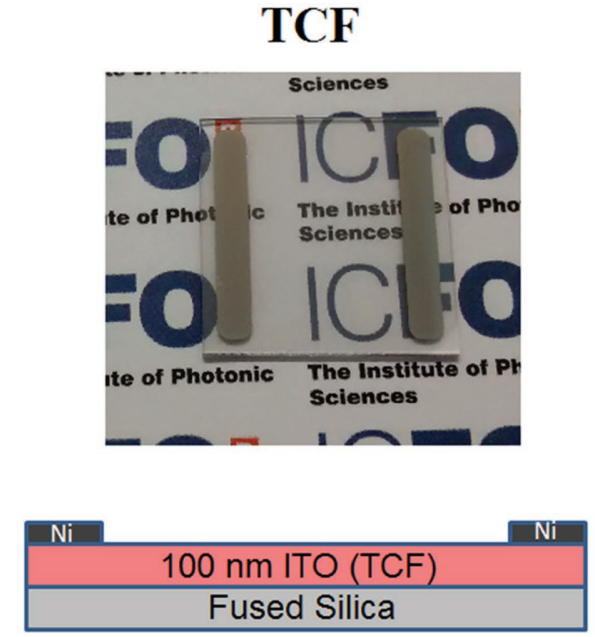

(a)

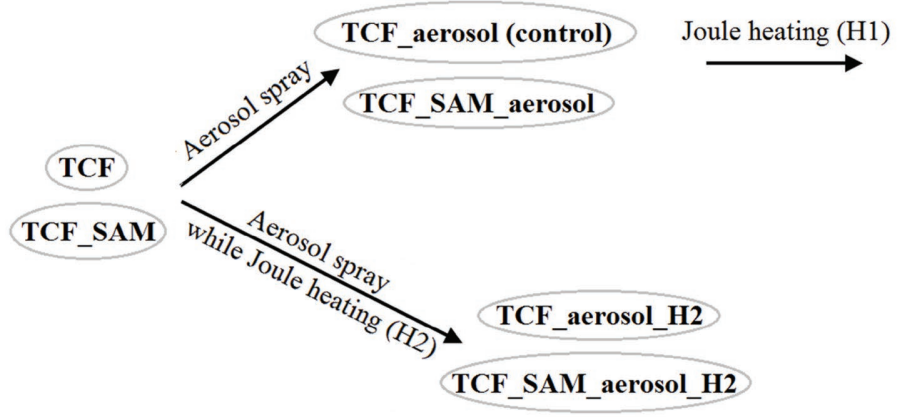

(c)

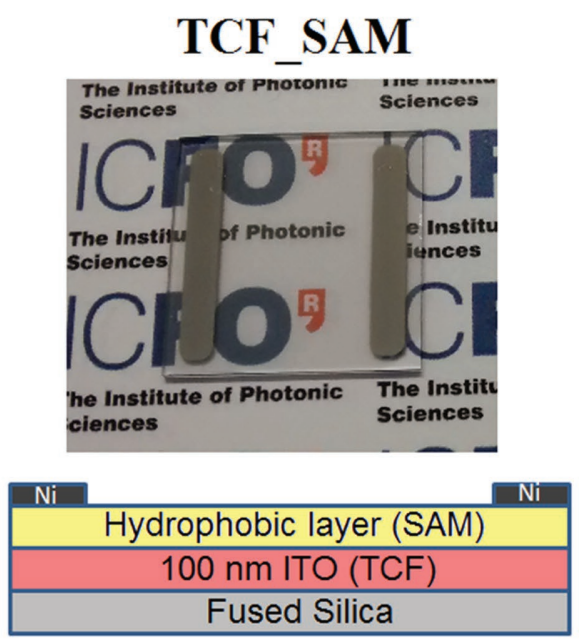

(b)

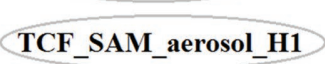

Figure 2. Images and cross-section schematics of the samples a) without SAM and b) with SAM. c) Experimental flowchart presenting the spraying and heating procedures. 
angle measurements were carried out for water and ink. For the samples without SAM and with SAM, CA of $5 \mu \mathrm{L}$ water drop was $78^{\circ}$ and $113^{\circ}$, respectively. For ink, three phase boundary moved after the droplet was placed onto the surface, hence CA at $t=0 \mathrm{~min}$ and $t=10 \mathrm{~min}$ were measured. CA of a $5 \mu \mathrm{L}$ ink drop on the samples without SAM and with SAM was $48^{\circ}$ and $67^{\circ}$ at $t=0 \mathrm{~min}, 13^{\circ}$ and $41^{\circ}$ at $t=10 \mathrm{~min}$, respectively (Figure S3 in the Supporting Information shows the measured droplet images). On the sample with SAM, sliding angles of 8 and $10 \mu \mathrm{L}$ water drops were measured to be $42^{\circ}$ and $22^{\circ}$, and for ink droplets $50^{\circ}$ and $35^{\circ}$, respectively. For the same volume of water and ink droplets, no sliding was observed on the sample without SAM. Effects of SAM on absorption, reflection, and transmission were negligible.

After the characterization of TCF Joule heating and SAM hydrophobicity, two different structures were considered for ink aerosol experiments. Samples with TCF alone (Figure 2a) and with TCF coated with SAM (Figure 2b) were sprayed using an ad hoc deposition system (see the Experimental Section for more details). Two different Joule heating modes were considered (Figure 2c): in the first one, the samples were heated after spraying the aerosol (heating mode 1, H1) while in the second, the samples were first heated, then maintained at a given temperature during spraying (heating mode 2, H2).

To characterize the ink aerosol on the samples, transmittance and light microscopy images of the samples were used. Transmittance (T\%) spectrums of the samples are given in Figure $3 \mathrm{a}$ and light microscopy images of the samples are presented in Figure 3b. From the light microscopy images, the percentage of the area covered by ink (PAC), number of particles per unit area $\left(N_{\mathrm{p}}\right.$ per $\left.\mathrm{mm}^{2}\right)$, and average size $\left(\mu \mathrm{m}^{2}\right)$ of the particles were extracted using image analysis, and are presented in Table 1. Clearly, the figure of merit for the sensor window is $T \%$. The more transparent the surface, the cleaner it is in terms of ink contamination. The light microscopy images were analyzed and quantitatively translated into values for further clarification of the effects of TCF and SAM on $T \%$.

T\% spectrum of the samples reveal that the sample with SAM and subjected to heating mode 2 (TCF_SAM_aerosol_H2) is the one less prone to ink aerosol contamination. The difference in optical transmittance between TCF_SAM_aerosol_H2 and the uncontaminated bare sample (TCF) is less than $1.5 \%$, for every wavelength in the measured spectrum. Individual effects of TCF (Joule heating) and SAM (hydrophobicity) can also be seen in Figure 5. First, for TCF, it

(b) can be seen that the Joule heating increases the transparency of the samples (both with and without SAM) contaminated with ink. As explained in the Experimental Section, two different heating modes were used. Among these, heating mode 2 (blue curves) outperforms heating mode 1 (red curves).

Second, for the effect of SAM, a significant difference between the samples with SAM and without SAM draws attention: there is a specific absorbance pattern for the samples without SAM, which is absent for the samples with SAM. It is reasonable to anticipate specific absorbance from ink, because it is used to provide a specific color, but the inks used for all
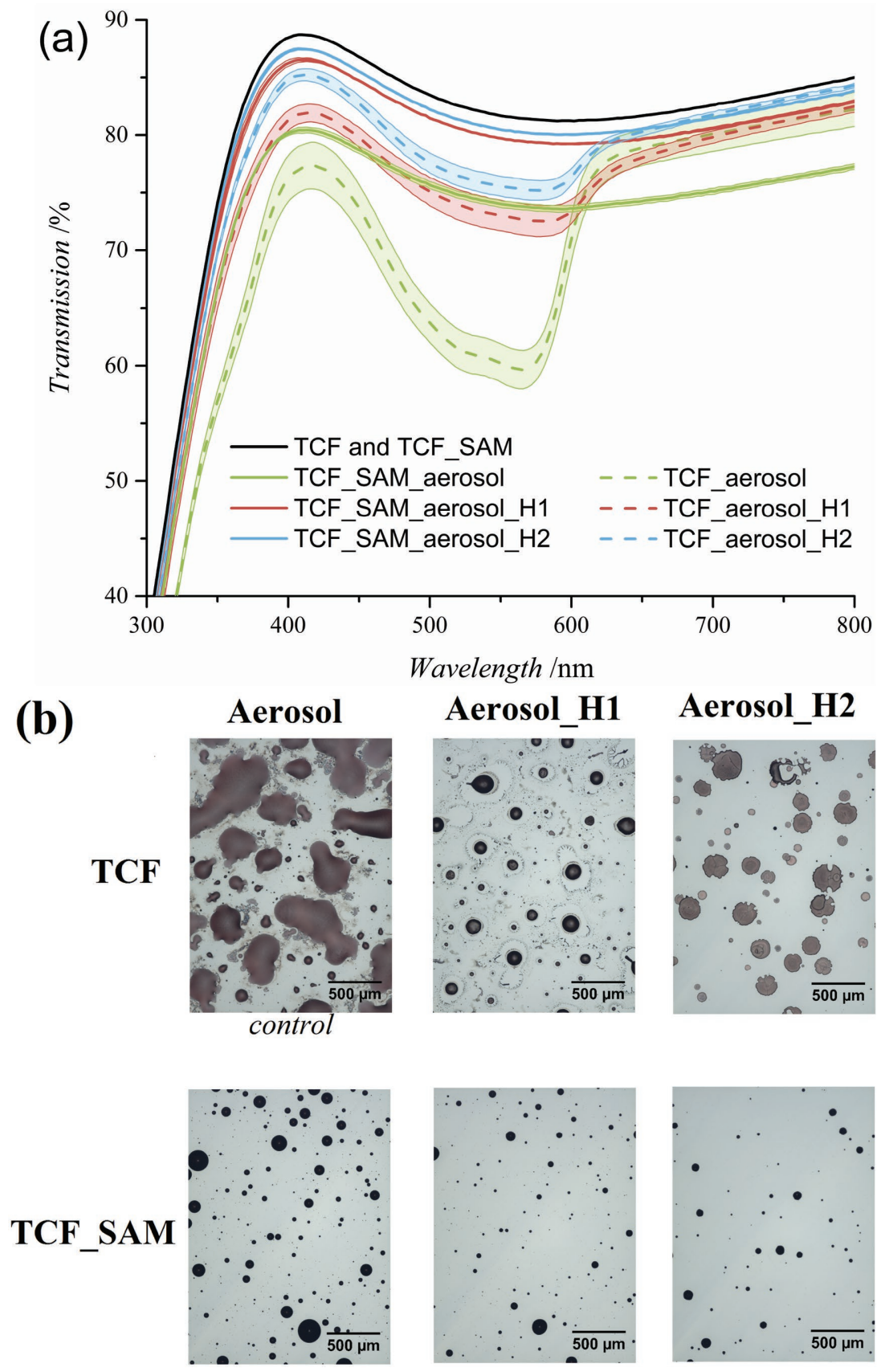

Figure 3. Characterization of the samples shown in the experimental flowchart. a) Optical transmittance spectrum. Fill area corresponds to the standard deviation. b) Light microscopy images. 
Table 1. Image processing results of the images shown in Figure 3b. Values indicated by plus or minus sign are standard deviation.

\begin{tabular}{lccc}
\hline Sample & $\begin{array}{c}\text { Area covered by ink (PAC) } \\
{[\%]}\end{array}$ & $\begin{array}{c}\text { Particle count } \\
{\left[N_{\mathrm{p}} \text { per } \mathrm{mm}^{2}\right]}\end{array}$ & $\begin{array}{c}\text { Average size } \\
{\left[\mu \mathrm{m}^{2}\right]}\end{array}$ \\
\hline TCF_aerosol (control) & $45.62 \pm 6.15$ & $245.89 \pm 93.30^{\mathrm{a})}$ & $2052.55 \pm 643.99$ \\
TCF_aerosol_H1 & $8.33 \pm 0.51$ & $475.40 \pm 26.58$ & $175.35 \pm 8.58$ \\
TCF_aerosol_H2 & $15.27 \pm 2.78$ & $27.55 \pm 4.45$ & $5663.90 \pm 1319.66$ \\
TCF_SAM_aerosol & $7.82 \pm 0.61$ & $93.54 \pm 6.38$ & $841.01 \pm 100.25$ \\
TCF_SAM_aerosol_H1 & $3.04 \pm 0.37$ & $90.06 \pm 4.52$ & $339.88 \pm 52.76$ \\
TCF_SAM_aerosol_H2 & $1.71 \pm 0.25$ & $19.28 \pm 2.09$ & $893.98 \pm 145.12$ \\
\hline
\end{tabular}

a)The bold values correspond to the case of glass substrates without SAM. In this case, the surface becomes very philic for the ink aerosols and the droplets tend to merge, making their distinction and counting difficult.

the experiments were the same and only in the case of samples without SAM, we do observe this wavelength-specific absorption. We believe that the observed difference is related to the height of the droplets. In the case of SAM, droplets had larger contact angle, thus larger average height, and acted as dark spots for the probing light, hence the wavelength independent decrease in transparency. Instead, in the case of samples without SAM, droplets were spread over a larger area, allowing light to go through them and consequently evidencing the colorant absorption fingerprint (Figure 4a). This is also consistent with the observed absorption spectrum of ink-water solution for varying ink concentration, from $0.1 \%$ to $100 \%$ (Figure $4 \mathrm{~b}$ ).
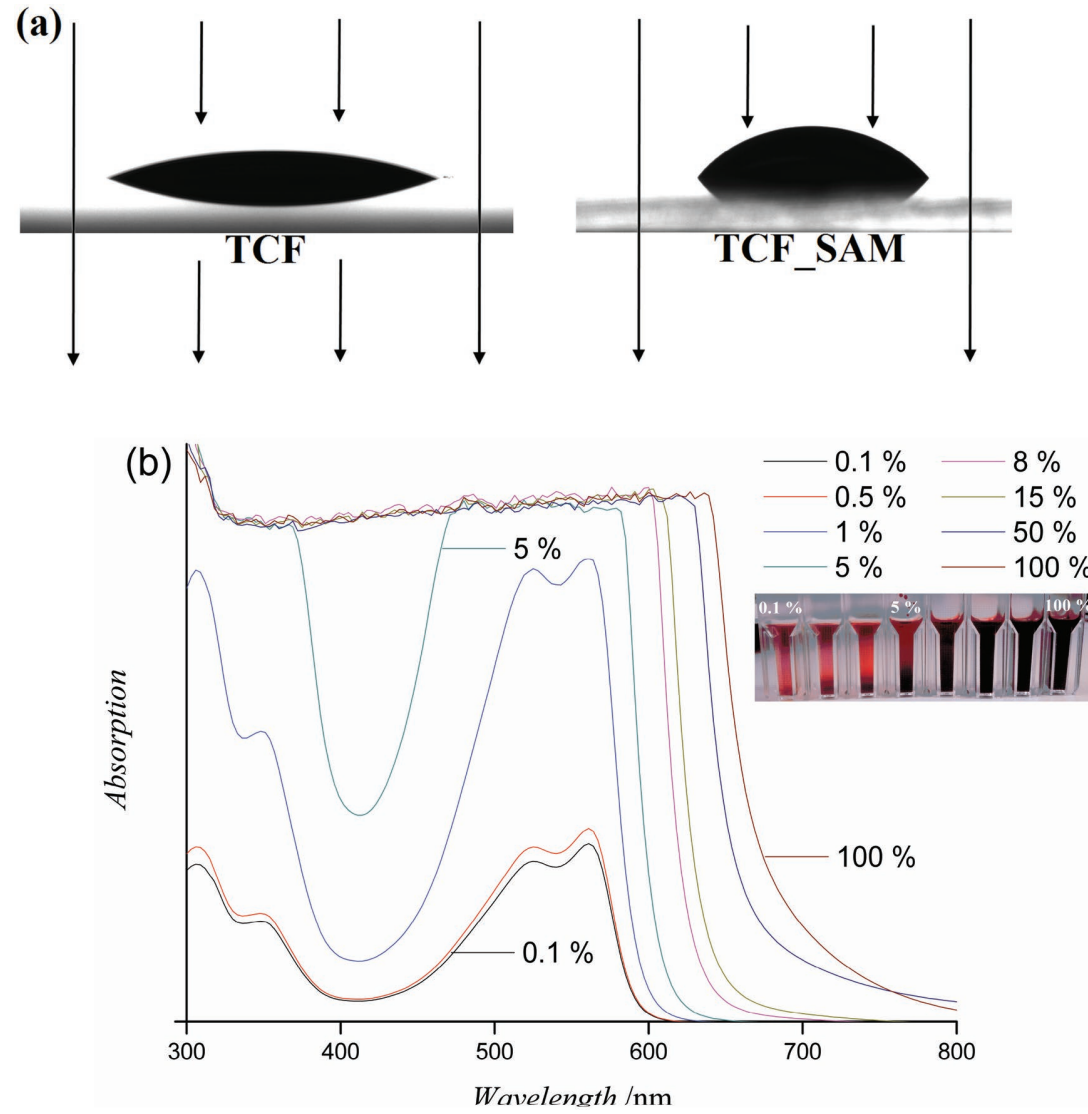

Figure 4. a) Image showing light-ink drop interaction depending on the contact angle. b) Absorption spectrum of ink-water solution for varying ink concentrations.
Above 5\% ink concentration, the absorption of the ink (colorant) was not any longer evident.

This makes the effect of SAM wavelength dependent. Comparing the dotted and solid curves of the same color in Figure 3a, one can see that below $600 \mathrm{~nm}$, samples with SAM are more transparent than samples without SAM. However, above $600 \mathrm{~nm}$, TCF_SAM_aerosol is less transparent than TCF_aerosol, above $600 \mathrm{~nm}$. For the samples with heating, SAM does not affect transparency significantly. This can be seen comparing TCF_aerosol_H1 to TCF_SAM_aerosol_H1 and TCF_aerosol_H2 to TCF_SAM_aerosol_H2, above $600 \mathrm{~nm}$.

Light microscopy images of the samples were taken and studied in order to understand the mechanisms behind the observed transmittance spectra. First, let us understand how heating increases transparency of contaminated samples. Upon heating TCF_SAM_aerosol, one obtains TCF_SAM_aerosol_H1. Comparing the light microscopy images of these two samples, one can see that almost all ink droplets remained on the surface, but they became smaller. This is confirmed by PAC value going down from $7.82 \% \pm 0.61 \%$ to $3.04 \% \pm 0.37 \%$, while the number of particles stays almost the same. This suggests that vehicle evaporates (ink consists of a colorant and a vehicle) and colorant stays on the surface. This can also be seen for the samples without SAM, comparing TCF_aerosol and TCF_aerosol_H1. One could observe some vehicle evaporating and colorant density increasing in the centers of ink droplets. To see the effect of heating modes, let us compare TCF_SAM_aerosol with TCF_ SAM_aerosol_H2. PAC value decreases from $7.82 \% \pm 0.61 \%$ to $1.71 \% \pm 0.25 \%$ and this time, number of particles does not stay the same, it goes down from $93.54 \pm 6.38$ to $19.28 \pm 2.09$. This might indicate that a large fraction of droplets evaporated or drifted away through convection before touching the surface. Comparing the samples without SAM supports this possibility. Evaporated droplet shape (colorant concentrated on the center leaving some vehicle around) which 
can be seen on TCF_aerosol_H1, cannot be seen on TCF_aerosol_H2. This also suggests that droplets might be evaporated before touching the surface. This explains why heating mode 2 outperforms heating mode 1 in transmittance measurements. Heating mode 1 removes vehicle from the surface, leaving the colorant behind. Heating mode 2 removes the vehicle and, in addition, prevents some fraction of ink reaching the surface, thus decreasing the colorant on the surface.

Effect of SAM can be seen by comparing the light microscopy images of TCF_aerosol and TCF_SAM_aerosol. As anticipated, droplet spreads more on the sample without SAM than on the sample with SAM. PAC value goes down from $45.62 \% \pm 6.15 \%$ to $7.82 \% \pm 0.61 \%$, respectively. In other words, sample with SAM has a larger uncontaminated area $(100 \%-(7.82 \% \pm 0.61 \%))$ compared to the sample without SAM $(100 \%-(45.62 \% \pm 6.15 \%))$. Hence, one might expect the samples with SAM to be more transparent compared to the samples without SAM, but as explained before, droplets on the sample with SAM are less transparent compared to the droplets on the sample without SAM due to the difference in average droplet height (Figure 4), and the transparency of the drops on the sample without SAM is greatly dependent on the wavelength due to the specific absorption of the ink. This interplay between opposite forces determines the wavelength dependency of the effect of SAM observed in transmittance measurements. Below $600 \mathrm{~nm}$, both drops on the samples TCF_SAM_aerosol and TCF_aerosol have similar opacity due to specific absorbance of the thinner drops on TCF_aerosol. Hence, PAC determines the transparency, lower PAC results in higher transmittances values. Above $600 \mathrm{~nm}$, drops on the sample with TCF_aerosol become almost transparent. This increases the transparency of TCF_aerosol above TCF_SAM_aerosol.

In addition, one can see that SAM also affects evaporation behavior of the droplets. On TCF_aerosol_H1, there are traces of vehicle around the remaining colorant, whereas the surrounding of the colorant on TCF_SAM_aerosol_H1 lacks visible trace of vehicle. This is because SAM reduces the stickiness of the surface.

Transparency evolution was also observed "in situ" (Figure S4 and Video S1, Supporting Information). Figure S4 (Supporting Information) shows how spraying decreased transparency of a sample with indium tin oxide (ITO) and SAM, while heating increased it, with a cleaning step of $50 \mathrm{~s}$.

The effect of heating and hydrophobicity was also demonstrated on the calibration sensor window (Figure 5). The window curvatures and nonuniform transparency did not allow performing a characterization as precise as with the fused silica. Nevertheless, the effect was visually evident and very similar to the detailed experiments reported above: ink aerosol content was significantly decreased, thanks to heating, hydrophobicity, and the combination of both effects.

\section{Conclusions}

We have investigated the self-cleaning effect of transparent surfaces covered with TCF and hydrophobic SAM, the former to induce Joule heating, against ink aerosol contamination. Despite the fact that Joule heating and hydrophobicity
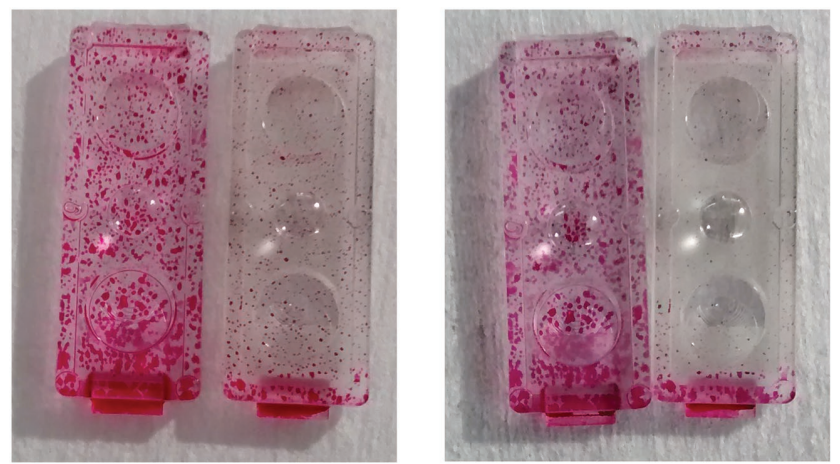

TCF_aerosol TCF_SAM_aerosol

TCF_aerosol_H1 TCF_SAM_aerosol_H1

Figure 5. Effect of hydrophobicity and heating on the actual sensor window.

(depending on the wavelength) individually reduced ink aerosol contamination, the experiments revealed that their combination was far more effective. Transmittance loss of the bare substrate (without hydrophobicity and Joule heating) sprayed with ink was around $10 \%$ on average and reaching $20 \%$ in the specific absorption range of the ink. Transmittance loss is reduced below $1.5 \%$ for the sprayed samples treated with both hydrophobicity and Joule heating. In addition, this treatment reduces the percentage of the area covered by ink from $45.62 \% \pm 6.15 \%$ to $1.71 \% \pm 0.25 \%$. The results obtained with glass substrates were extended to the plastic window of an optical calibration sensor widely used in commercial inkjet printers, thus demonstrating the potential of the proposed approach to prevent and reduce aerosol contamination in real world applications.

\section{Experimental Section}

Joule Heating: The TCF for surface Joule heating was a $100 \mathrm{~nm}$ thick ITO film deposited onto $25 \times 25 \mathrm{~mm}^{2}$ fused silica substrates using sputtering (AJA International ATC Orion $8 \mathrm{HV}$ ). After cleaning the substrate using acetone and methanol in an ultrasonic bath, sputtering of the ITO was carried out. Substrate-target distance was set to $30 \mathrm{~cm}$. When the base pressure reached $0.01 \mathrm{mTorr}, \operatorname{Ar}(20 \mathrm{sccm})$ and $\mathrm{O}_{2}$ (1 sccm), deposition precursor gases were introduced into the system and the deposition pressure reached 2 mTorr. After deposition of the TCF, nickel stripe electrical contacts were deposited on the ITO to achieve a uniform current (heating) over the entire transparent surface (Figure 2a). A power supply (AIM-TTI Instruments EL302R) was used to apply a constant electrical current. The temperature distribution over the heated surface was measured with an IR camera (Keysight Technologies U5855A TruelR Thermal Imager). The average temperature was kept constant between 80 and $85{ }^{\circ} \mathrm{C}$ for all experiments, by applying direct current of $0.27 \mathrm{~A}$ and $9 \mathrm{~V}$.

Hydrophobicity: Samples were coated with a SAM, alkoxysilane functional perfluoropolyether hybrid polymer from Dow Corning (2634 Coating). The SAM was initially diluted to $0.1 \%$ with a fluorinated solvent (Novec HFE 7200). Substrates were dipped into the solution for $3 \mathrm{~min}$ and dried at $50{ }^{\circ} \mathrm{C}$ for $1 \mathrm{~h}$. Finally, they were rinsed in Novec HFE7200 solvent. Contact angle and sliding angles for water and ink droplets were measured using Krüss GmbH DSA100 drop shape analyzer.

Aerosol Deposition: This study required a repeatable aerosol generation and deposition system, capable of generating aerosol particles with sizes similar to the ones generated inside the printer. Early studies involving aerosols generally used custom-built aerosol generation systems and nowadays, variety of commercial aerosol generators exist. ${ }^{[1]}$ In this study, a spray setup was built for aerosol deposition, comprising an 
Agar Scientific Glass microspray device. For repeatability, a purge valve and pressure controller were used instead of hand squeeze ball pump. Holders were used to fix the microspray and the target substrate. Each sample was sprayed for $48 \mathrm{~s}$. Electrical wiring enabled the application of electrical current to the sample while the latter was in the setup. Images of the spray setup can be seen in Figure S5 in the Supporting Information.

Characterization of the Ink Aerosol Contamination: A UV-vis spectrophotometer (Perkin Elmer Lambda 950) was used to measure the transmittance (T\%) of the samples, which could be considered a reliable quantification of the overall aerosol contamination on the samples. Optical microscopy was used to analyze the ink aerosol distribution on the substrate. The images were processed to measure the percentage of area covered by the ink aerosol (PAC, \%), number of particles per unit area $\left(N_{p}\right.$ per $\left.\mathrm{mm}^{2}\right)$, and average size of the particles. The entire experiments and measurements were repeated three times on each sample. Each time (i.e., each spray) five images were taken from different points of the sample. In addition, a USB microscope (XCSOURCE TE071) was placed inside the spraying setup to record a video of the sample behavior and a specific optical setup was designed to measure transmittance during the entire experiment.

\section{Supporting Information}

Supporting Information is available from the Wiley Online Library or from the author.

\section{Acknowledgements}

The authors would like to thank Carles Castellsague, Xavier Bruch, Jordi Ferran, Joan Saez from HP Inc. for fruitful discussions. This work had been performed with the support of the Secretary of Universities and Research, Department of Business and Knowledge (DECO), Government of Catalonia. In addition, the authors acknowledge financial support from the Fundació Privada Cellex, the AGAUR 2014 SGR 1623, the Spanish Ministry of Economy and Competitiveness (MINECO), through the "Severo Ochoa" Programme for Centres of Excellence in R\&D (SEV-2015-0522) and the "Fondo Europeo de Desarrollo Regional" (FEDER) through grant TEC2013-46168-R.

Received: June 7, 2016

Revised: September 6, 2016

Published online: October 26, 2016

[1] P. Calvert, Chem. Mater. 2001, 13, 3299.

[2] G. Cummins, M. P. Y. Desmulliez, Circuit World 2012, 38, 193.

[3] J. T. Delaney, P. J. Smith, U. S. Schubert, Soft Matter 2009, 5, 4866.

[4] M. Singh, H. M. Haverinen, P. Dhagat, G. E. Jabbour, Adv. Mater. 2010, 22, 673.

[5] T. Xu, J. Jin, C. Gregory, J. J. Hickman, T. Boland, Biomaterials 2005, 26, 93.

[6] S. D. Hoath, Fundamentals of Inkjet Printing: The Science of Inkjet and Droplets, Wiley-VCH, Weinheim, Germany 2016.

[7] C. L. Holstun, S. D. Asakawa (Hewlett-Packard Development Company, L.P.), US7066564 B2, 2006

[8] M. Boleda, R. R. Giles, P. D. Gast (Hewlett-Packard Company), US6203152 B1, 2001.

[9] P. Ragesh, V. Anand Ganesh, S. V. Nair, A. S. Nair, J. Mater. Chem. A 2014, 2, 14773

[10] H. M. Lemire, K. A. Peterson, M. S. Breslau, K. D. Singer, I. T. Martin, R. H. French, MRS Proceedings 2013, 1537, mrss13-1537.

[11] R. N. Udey, A. D. Jones, G. R. Farquar, Aerosol Sci. Technol. 2013, 47, 361. 